\title{
MÉTODO DE QUANTIFICAÇÃO DA COBERTURA VERDE FRACIONADA EM CULTIVARES DE AMENDOIM COM INTERFERÊNCIA DE PLANTA DANINHA
}

Pablo Nascimento de Oliveira França' ${ }^{1}$; Willians César Carrega ${ }^{2}$; Ana Julia Borges Lima ${ }^{3}$; Bruno Saravali Silveira ${ }^{4}$; Jose Davi Phelipe ${ }^{5}$; Pedro Luis da Costa Aguiar Alves ${ }^{6}$

${ }^{1}$ Mestrando em Agronomia (Ciência do Solo), FCAV/UNESP, Jaboticabal, SP, pnof@hotmail.com; ²Doutor em Agronomia (Produção Vegetal), FCAV/UNESP, Jaboticabal, SP; ${ }^{3}$ Graduando em Agronomia, FCAV/UNESP, Jaboticabal, SP; ${ }^{4}$ Graduando em Agronomia, FCAV/UNESP, Jaboticabal, SP; ${ }^{5}$ Graduando em Agronomia, FCAV/UNESP, Jaboticabal, SP; ${ }^{6}$ Docente da FCAV/UNESP, Jaboticabal, SP.

RESUMO: O uso de aplicativos tem sido uma opção viável para auxiliar no campo de produção de várias culturas. Por isso, este trabalho teve como objetivo avaliar a eficiência do aplicativo Canopeo ${ }^{\odot}$ em estimar a supressão imposta pela presença da mucuna-preta (Mucuna aterrima) em cultivares de amendoim, com intuito de quantificar de forma fracionada a porcentagem de biomassa das plantas. Realizou-se um experimento em delineamento em blocos casualizados, tendo como tratamentos cinco cultivares (IAC 503, IAC 505, IAC OL3, IAC OL4 e IAC OL5) com e sem a presença da mucunapreta, com 4 repetições. Para quantificar a cobertura verde fracionada, utilizou-se o aplicativo Canopeo $^{\odot}$, que baseia-se na leitura de imagens por colorimetria, oferecendo resultados in situ precisos e com menor tempo. A redução da porcentagem de cobertura verdedo amendoim em convivência com a mucuna-preta foi de $70 \%, 84 \%, 84 \%, 88 \%$ e $89 \%$ para as cultivares IAC 503, IAC 505, IAC OL3, IAC OL4 e IAC OL5, respectivamente.Concluímos que o aplicativo Canopeo ${ }^{\circ}$ v. 2.0 é eficiente para determinar a supressão imposta pela mucuna-preta para as cultivares de amendoim estudadas. No entanto, há necessidades de novos estudos com o aplicativo, utilizando diferentes espécies de plantas daninhas, correlacionando a produtividade do amendoim com a redução da cobertura vegetal.

Palavras-chave: Arachis hypogaea L., Canopeo ${ }^{\circledR}$, Monitoramento, Mucuna aterrima.

\section{INTRODUÇÃO}

A interferência causada pala competição de plantas daninhas com a cultura do amendoim pode causar severasreduçõesno rendimento (AGOSTINHO et al., 2006; YAMAUTI et al., 2010). As plantas daninhas competem diretamente por recursos vitais com a cultura como, água, luz e nutrientes (GIMENES et al., 2011). Devido a essa importância é necessário o constante monitoramento dessas áreas para o controle de possíveis danos na produção.

Entre as plantas daninhas de grande preocupação, a mucuna-preta (Mucuna aterrima) foi inicialmente implantada em áreas de reforma de canavial, visando a melhoria do solo. Entretanto, com o passar do tempo e devido principalmente a dormência de suas sementes, sua ocorrência passou a ser um problemaem áreas de produção de cana-de-açúcar, acarretando perdas significativas no potencial produtivo 
das culturas (BRESSANIN et al. 2016). Além disso, essa espécie apresenta grande quantidade de massa vegetativa, suprimindo na maioria das vezes a cultura de interesse produtivo (DE SOUZA et al., 2015), entre elas, a do amendoim, que comumente é semeado em áreas de reforma de canaviais.

Entre as inovações tecnológicas, o uso de aplicativos para auxiliar na agricultura vem sendo adotado em muitas culturas. Em amendoim, já existem aplicativos para avaliar a maturação das vagens, estimar as perdas na produtividade e auxiliar na identificação de doenças e pragas, entre outros. Pesquisadores de duas Universidades Americanas, lançaram um aplicativo intitulado Canopeo $^{(}$, que visaquantificar a cobertura de dossel da cultura, tendo como base a leitura de imagens da vegetação (PATRIGNANI; OCHSNER, 2015). Sua operação é baseada em colorimetria, oferecendo resultados in situ precisos e com menor tempo. É útil no monitoramento do crescimento das culturas, quantificação do efeito de fatores ambientais como granizo, neve, estresse hídrico, recomendações de fertilizantes e estimativa da interceptação de luz.

O Canopeo ${ }^{\odot}$ já foi utilizado para avalições em culturas como milho, sorgo, batata, alface, capim-bermuda, família de Panicum e hortaliças, classificando corretamente até $90 \%$ dos pixels quando comparado ao outros métodos de avaliação como SamplePoint (PATRIGNANI; OCHSNER, 2015; YELLAREDDYGARI; GUDMESTAD, 2017). Sua praticidade e rapidez em gerar dados pode ser utilizado como ferramenta para auxiliar no monitoramento em áreas de produção de muitas culturas, inclusive do amendoim. $\mathrm{O}$ Canopeo ${ }^{\odot}$ pode ser uma ferramenta viável para quantificar o impacto da interferência em decorrência da competição de espécies daninhas na produtividade da cultura. Diante disso, este trabalho teve como objetivo avaliar a eficiência do aplicativo Canopeo ${ }^{\circledR} \mathrm{em}$ estimar a supressão imposta pela presença da mucuna-preta (Mucuna aterrima) em cultivares de amendoim, com intuito de quantificar de forma fracionada a porcentagem de biomassa das plantas.

\section{MATERIAL E METODOS}

O experimento foi conduzido no Laboratório de Plantas Daninhas (LAPDA), localizado na Universidade Estadual Paulista "Júlio de Mesquita Filho", Câmpus de Jaboticabal, São Paulo. O delineamento utilizado foi em blocos casualizados, tendo como tratamentos cinco cultivares (IAC 503, IAC 505, IAC OL3, IAC OL4 e IAC OL5) com e sem a presença da mucuna-preta, em 3 repetições.As sementes das cinco cultivares de amendoim foram previamente tratadas com o fungicida thiram (Vitavax ${ }^{\circledR}$-Thiram $200 \mathrm{SC}, 250 \mathrm{~mL}$ do p.c. $100 \mathrm{~kg}^{-1}$ ) para evitar a interferência de patógenos.

Em caixa fixas ao solo com medidas de $1 \mathrm{~m}^{2} \mathrm{x}$ 2,5 $\mathrm{m}$ de profundidade, foram semeadas em média 20 sementes de amendoim por caixa e 10 sementes de mucuna-preta $/ \mathrm{m}^{2}$. Após a emergência realizou-se desbaste, deixando 12 plantas de amendoim e 5 plantas de mucuna-preta por parcela. Durante o período experimental foram realizados acompanhamentos semanais de possíveis doenças e pragas, bem como seu controle por meio de pulverizações, com aplicação dos defensivos 
recomendados, quando necessário. Também, manteve-se as plantas hidratadas de acordo com a capacidade de campo.

Para avalição da área foliar, utilizou-se o aplicativo Canopeo ${ }^{\circ} \mathrm{v} 2.0$, instalado em um smartphone, seguindo as recomendações dos desenvolvedores do software (PATRIGNANI; OCHSNER, 2015), incluindo a calibração para a cultura, altura e datas de semeadura.O aplicativo possui a capacidade de acessar a câmera do smartphonepara estimar a cobertura do dossel verde estimando os valores em porcentagem. Durante as avaliações, as imagens foram capturadasà aproximadamente $0,60 \mathrm{~m}$ de altura, seguindo metodologia proposta por Yellareddygari; Gudmestad (2017). Além do uso do aplicativo, realizou-se avaliações de altura das plantas, comprimento dos ramos primários, número de ramos primários e número de vagens por parcela.

Os resultados obtidos foram submetidos a análise de variância pelo teste $\mathrm{F}$ e as médias comparadas pelo teste de Tukey a 5\% de probabilidade, utilizando o software Agrostat (BARBOSA; MALDONADO JUNIOR, 2015).

\section{RESULTADOS E DISCUSSÃO}

No presente estudo, avaliando a porcentagem de cobertura verde (PCV) das cinco cultivares de amendoim ao final do ciclo da cultura, observou-se redução no dossel de forma significativa para todos os cultivares. A redução da PCV foi de $70 \%, 84 \%, 84 \%, 88 \%$ e $89 \%$ para as cultivares IAC 503, IAC 505, IAC OL3, IAC OL4 e IAC OL5, respectivamente (Figura 1). Esse efeito negativo, demonstra que há a necessidade de controlar essa espécie rapidamente, pois a mucuna-preta tem grande potencial competitivo com a cultura do amendoim, devido ao efeito supressor causado por essa espécie daninha. Reduções na biomassa vegetativa do amendoim, em decorrência de plantas daninhas causando sombreamento e sufocamento da cultura, também foi constatado por Korav et al. (2018). Bressanin et al. (2016) constataram redução de 50\% no rendimento da cana-de-açúcar em convivência com a mucuna-preta. 
IAC 503

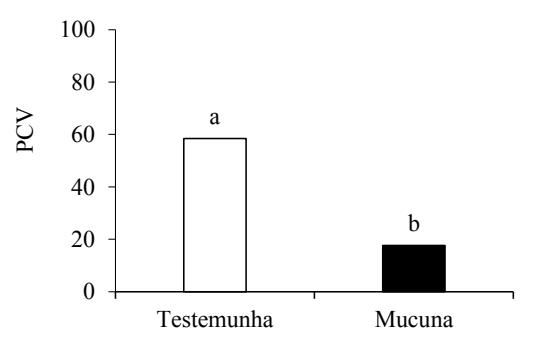

IAC OL3

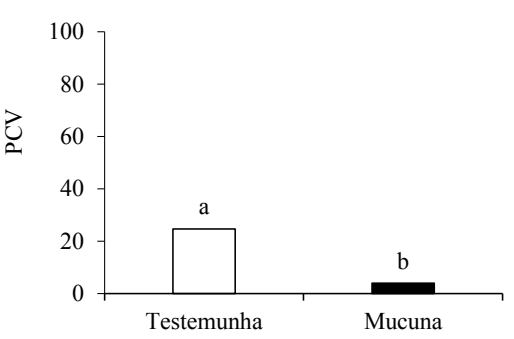

IAC OL4

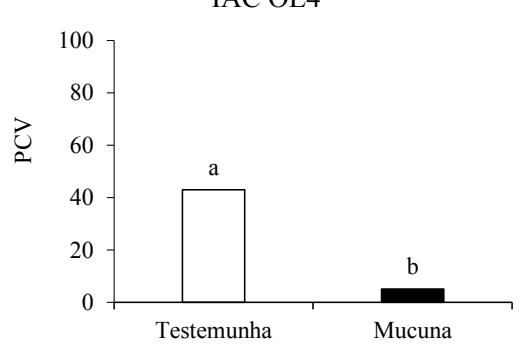

IAC 505

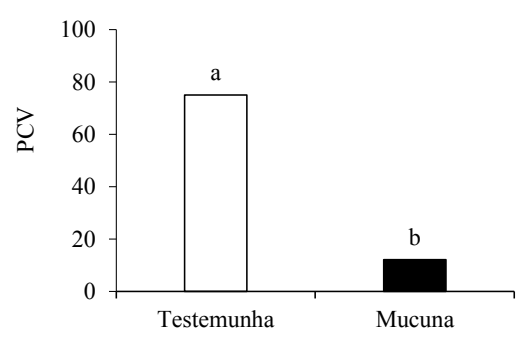

IAC OL5

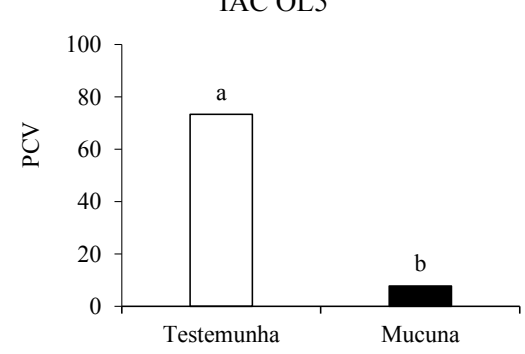

Figura 1. Porcentagem de cobertura verde (PCV) de cultivares de amendoim sob interferência da mucuna-preta.

As reduções na biomassa vegetativa observadas com uso do aplicativo (Figura 1 e 2), coincidem com as reduções constatadas para a altura das plantas, comprimento dos ramos primários, número de ramos primários e número de vagens (Figura 3), indicando que a interferência ocasionada pela convivência com a mucuna-preta afeta diretamente o crescimento e desenvolvimento das plantas, reduzindo a biomassa vegetativa e promovendo reduções significativas para a produtividade de vagens do amendoim. $\mathrm{O}$ uso do Canopeo $\odot$ foi uma ferramenta útil para quantificar e estimar a porcentagem de cobertura do dossel em amendoim. A eficiência do aplicativo corrobora os resultados de pesquisas realizadas para outras culturas (BÜCHI et al. 2018; O’BRIEN et al., 2018). O uso do Canopeo® já está sendo utilizado em áreas agrícolas, sendo utilizado no manejo integrado de plantas daninhas em arroz irrigado (SENGXUA et al., 2018) e, com base em nossos resultados, esse aplicativo pode ser uma ferramenta promissora em áreas de produção de amendoim.

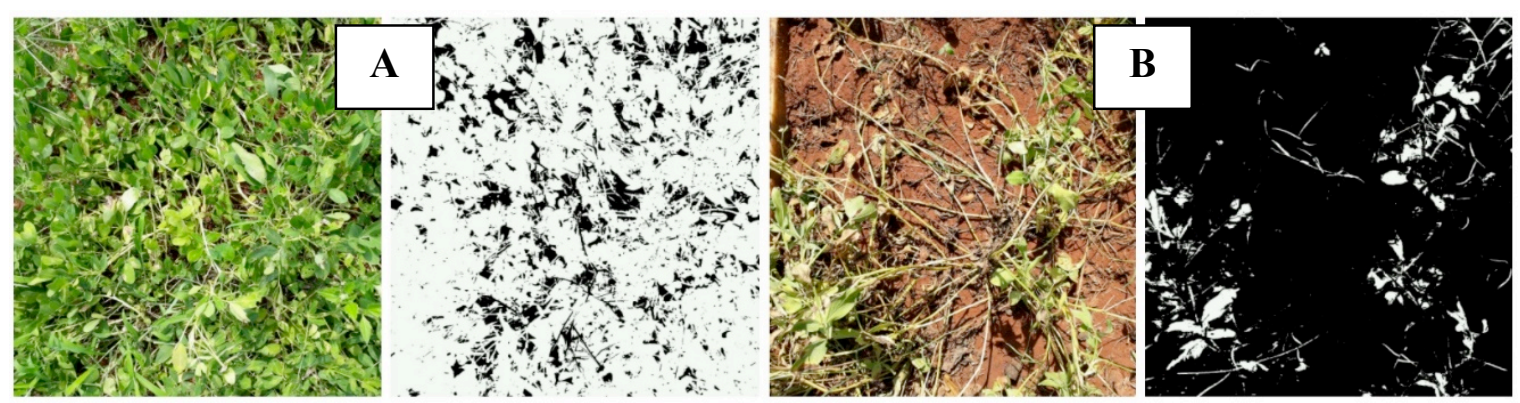

Figura 2. Cobertura do dosselbaseada em colorimetria e estimado pelo aplicativo Canopeo ${ }^{\odot}$ v. 2.0. A, sem presença de mucuna-preta; B, amendoim sob interferência de mucuna-preta. 

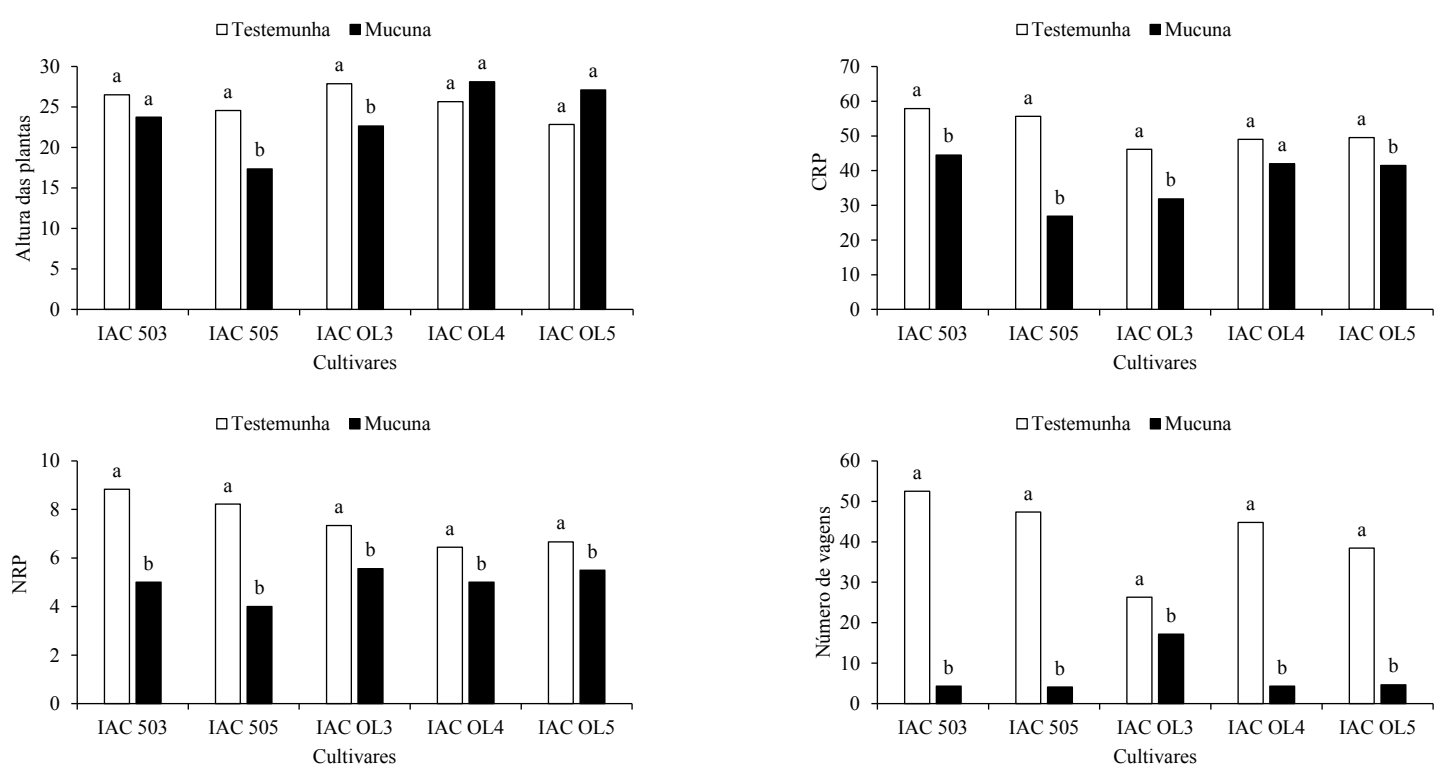

Figura 3. Altura de plantas, comprimento do ramo primário mais desenvolvido (CRP), número de ramos primários (NRP) e número de vagens de cultivares de amendoim sob interferência da mucunapreta.

\section{CONCLUSÃO}

Concluímos que o aplicativo Canopeo $(\mathcal{C}$ v. 2.0 é eficiente para determinar a supressão imposta pela mucuna-preta para as cultivares de amendoim IAC 503, IAC 505, IAC OL3, IAC OL4 e IAC OL5. No entanto, há necessidades de novos estudos com esse aplicativo, utilizando diferentes espécies de plantas daninhas, correlacionando a produtividade do amendoim com a redução da cobertura vegetal.

\section{REFERÊNCIAS BIBLIOGRÁFICAS}

Agostinho, F. H.; GRAVEnA, R.; AlveS, P. L. C. A.; SAlgado, T. P.; MATTOS, E. D. The effect of cultivar on critical periods of weed control in peanuts. Peanut Science, v.33, n.1, p.29-35, 2006.

BARBOSA, J. C.; MALDONADO JUNIOR, W. 2015. AgroEstat - sistema para análises Estatísticas de ensaios agronômicos. Jaboticabal: FCAV/UNESP. 396p.

BRESSANIN, F.N.; JAYME NETO, N.; NEPOMUCENO, M.P.; ALVES, P.L.C.A.; CARREGA, W.C. Interference periods of velvet bean in sugarcane. Ciência Rural, v.46, n.8, p.1329-1337, 2016. BÜCHI, L.; WENDLING, M.; MOULY, P.; CHARLES, R. Comparison of visual assessment anddigital image analysis for canopy cover estimation. Agronomy Journal, v.110, n.4, p.1289-1295, 2018 .

DE SOUZA, G. C.; TIMOSSI, P. C.; DA COSTA NETTO, A. P.; RODRIGUES, M. J.; FARIA FILHO, L. A. Germinação de sementes de mucuna-preta submetidas a diferentes períodos de armazenamento. Revista Brasileira de Energia Renováveis, v.4, n.1, p.72-83, 2015. 
Gimenes, M. J.; POGETTO, M. H. F. A. D.; PRADO, E. P.; CHRISTOVAM, R. S.; COSTA, S. Í. A.; SOUZA, E. F. C. Interferência de Brachiaria Ruziziensis sobre plantas daninhas em sistema de consórcio com milho. Semina: Ciências Agrárias, v.32, n.3, p.931-938, 2011.

KORAV, S.; RAM, V.; RAY, L. I.; KRISHNAPPA, R.; SINGH, N. J.; PREMARADHYA, N. Weed Pressure on Growth and Yield of Groundnut (Arachis hypogaea L.) in Meghalaya, India. International Journal of Current Microbiology and Applied Sciences, v.7, n.3, p.2852-2858, 2018. O’BRIEN, P. L.; ACHARYA, U.; ALGHAMDI, R.; NIAGHI, A. R.; SANYAL, D.; WIRTZ, J.; DAIGH, A. L. M.; DESUTTER, T. M. Hydromulch Application to Bare Soil: Soil Temperature Dynamics and Evaporative Fluxes. Agricultural \& Environmental Letters, v.3, n.1, p.1-5, 2018.

PATRIGNANI, A.; OCHSNER, T.E., 2015. Canopeo: A powerful new tool for measuring fractional green canopy cover. Agronomy Journal, v.107, n.6, p.2312-2320, 2015.

SENGXUA, P.; JACKSON, T.; SIMALI, P.; VIAL, L. K.; DOUANGBOUPHA, K.; CLARKE, E.; HARNPICHITVITAYA, D.; WADE, L. J. Integrated nutrient-weed management under mechanised dry direct seeding (dds) is essential for sustained smallholder adoption in rainfed lowland rice (Oryza sativa). Experimental Agriculture, p.1-17, 2018.

YAMAUTI, M. S.; ALVES, P. L. C. A.; NEPOMUCENO, M.; MARTINS, J. V. F. Adubação e o período anterior à interferência das plantas daninhas na cultura do amendoim. Planta Daninha, v.28, p.1031-1039, 2010.

YELLAREDDYGARI, S. K. R.; GUDMESTAD, N. C. Bland-Altman comparison of two methods for assessing severity of Verticillium wilt of potato. Crop Protection, v.101, p. 68-75, 2017. 